\title{
Romanian Questionnaire to Assess
} the Prevalence of Occupational Hand
Eczema among Healthcare Providers

\author{
Anca E. Chiriac', Doina Azoicai², Anca Chiriac ${ }^{3,4,5}$, Adrian Naznean ${ }^{6}$, Francesca Larese Filon 7 , \\ Simona Roxana Georgescu ${ }^{8}$, Liliana Foia ${ }^{9}$, Cristian Podoleanu'10, Simona Stolnicu11 \\ 1 "Grigore T. Popa” University of Medicine and Pharmacy, Iași, Romania \\ 2 Department of Epidemiology, "Grigore T. Popa” University of Medicine and Pharmacy, lași, Romania \\ 3 Nicolina Medical Center, Department of Dermatology, lași, Romania \\ ${ }^{4}$ Apollonia University, lași, Romania \\ 5 "Petru Poni" Research Institute, Romanian Academy, Iași, Romania \\ 6 Department of Foreign Languages, University of Medicine and Pharmacy, Tîrgu Mureș, Romania \\ 7 Unit of Occupational Medicine, University of Trieste, Italy \\ 8 Department of Dermatology, "Carol Davila” University of Medicine and Pharmacy, Bucharest, Romania \\ 9 Department of Biochemistry, "Grigore T. Popa” University of Medicine and Pharmacy, lași, Romania \\ 10 Department of Internal Medicine IV, University of Medicine and Pharmacy, Tîrgu Mureș, Romania \\ ${ }^{11}$ Department of Pathology, University of Medicine and Pharmacy, Tîrgu Mureș, Romania
}

\section{CORRESPONDENCE \\ Cristian Podoleanu \\ Str. Gheorghe Marinescu nr. 1 \\ 540139 Tîrgu Mureș, Romania \\ Tel: +40 265215133 \\ E-mail: podoleanu@me.com}

\section{ARTICLE HISTORY}

Received: 28 October, 2016

Accepted: 10 November, 2016

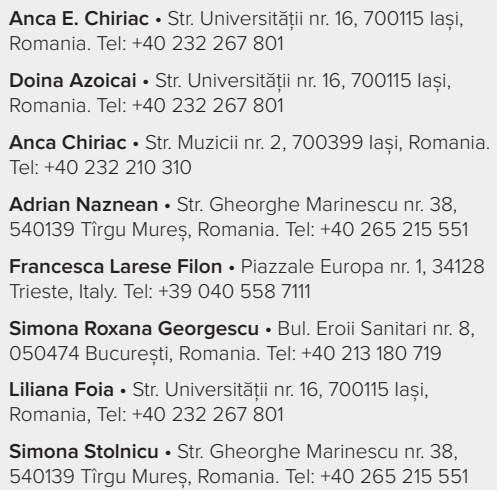

\begin{abstract}
Occupational skin diseases have an unknown prevalence in Romania, although they are considered the most frequent occupational diseases reported in Western European countries. Self-reported hand eczema among healthcare providers by questionnaire aims to estimate the prevalence of work-related hand eczema and associated risk factors in hospitals and outpatient units in Romania. The aim of this study is to discuss and to validate a questionnaire for surveying work-related skin diseases and exposure among healthcare providers.
\end{abstract}

Keywords: hand eczema, healthcare professionals, questionnaire

Occupational skin diseases should be a priority for public health because they represent up to $30 \%$ of occupational diseases. ${ }^{1}$ The recognition of occupational skin diseases is important for improving prevention and patient management, as their economic and social impact on each individual and the society is significant. Occupational hand eczema among healthcare providers has a great impact on their quality of life and, consequently, on their professional activity.

The purpose of this study is to develop a standardized questionnaire that can be used as a tool for self-reporting, diagnosing and monitoring the prevalence of hand dermatitis among healthcare providers in Romania. 


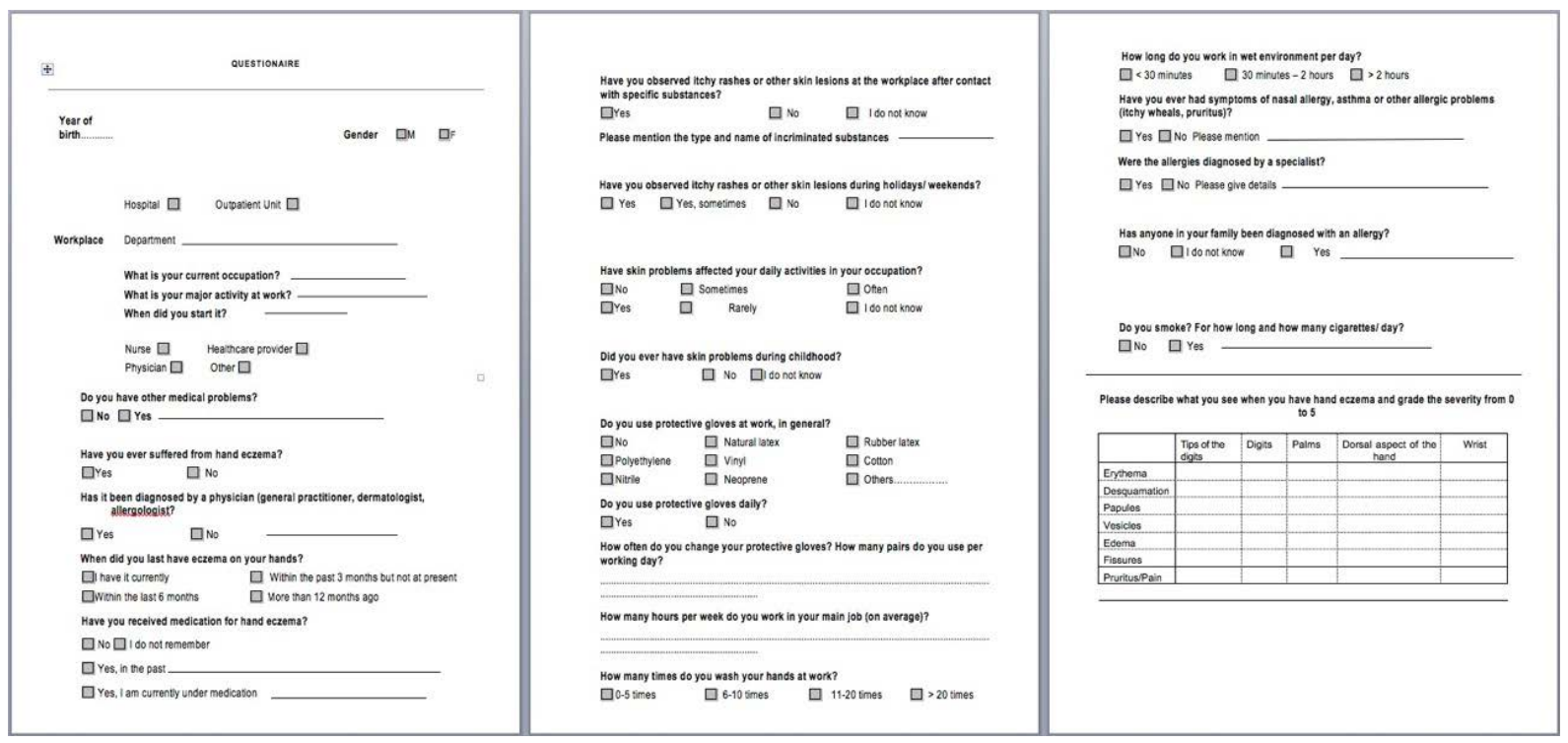

FIGURE 1. The proposed self-reported questionnaire

The questionnaire was compiled after a validated Italian work-related questionnaire, which was translated to Romanian, and its target group is represented by healthcare professionals from different medical fields. It is a type of self-report of hand eczema/dermatitis to estimate the prevalence of the disease in the target group. The questionnaire is simple, short (three pages), easy to understand and to complete, it requires "yes" or "no" answers to specific questions, it is anonymous and focused on the clinical aspects related to direct work exposure (Figure 1). Details regarding contact with work-related aggravating factors are also assessed: contact with chemical agents (detergents, disinfectants, soap, liquid soap, shampoo, and other personal hygiene products or skin cleansers), use of latex or other types of gloves (plastic, cotton, natural or synthetic rubber). Other relevant exposures, such as the frequency of hand washing, are also surveyed. The medical history of the surveyed employees is also relevant based on the atopic or allergic background of each individual, the presence of comorbidities, chronic treatments or other exposures such as UV radiation. ${ }^{2-4}$

Based on the results of the study, early dermatological interventions and specific education regarding personal skin protection can be initiated. Moreover, the study will enable the quantification of allergens and/or irritant agents and their confirmation by patch testing.

Screening for predisposing factors (especially atopic or genetic predisposition) can be evaluated, and in case of susceptible individuals career counseling and targeted prevention strategies can be provided.

The questionnaire allows the identification of subjects who are susceptible to develop occupational skin diseases, the recognition of work-related risk factors, and it should represent the first step in the elaboration of preventive measures concerning specific occupations and risk profiles.

Underestimation of the prevalence of occupational hand eczema can be expected from self-reporting due to numerous factors: lack of understanding of all questions, fear to provide honest answers that can affect daily activity, unwillingness to acknowledge the existence of symptoms, lack of compliance or knowledge.

This is the first self-reported questionnaire in Romania that tries to estimate the prevalence of irritant and allergic contact dermatitis of the hands among employees in the specific sector of healthcare services.

\section{ACKNOWLEDGEMENT}

The authors are grateful for networking support from the COST Action TD1206 "StanDerm".

\section{REFERENCES}

1. Occupational skin diseases and dermal exposure in the European Union (EU-25): policy and practice overview. Available online from: https://osha.europa.eu/en/tools-and-publications/publications/reports/ TE7007049ENC_skin_diseases

2. Susitaival P, Flyvholm MA, Meding B, et al. Nordic Occupational Skin Questionnaire (NOSQ-2002): a new tool for surveying occupational skin diseases and exposure. Contact Dermatitis. 2003;49:70-76.

3. Carlsson A, Ganemo A, Anderson CD, Meding B, Stenberg B, Svensson A. Scoring of hand eczema: good agreement between patients and dermatological staff. Br J Dermatol. 2011;165:123-128.

4. Pesonen M, Jolanki R, LareseFilon F, et al. Patch test results of the European baseline series among patients with occupational contact dermatitis across Europe - analyses of the European Surveillance System on Contact Allergy network, 2002-2010. Contact Dermatitis. 2015;72:154-163 